\title{
Food, youth and the Mediterranean diet in Spain. Development of KIDMED, Mediterranean Diet Quality Index in children and adolescents
}

\author{
Lluís Serra-Majem ${ }^{1,2, *}$, Lourdes Ribas ${ }^{2}$, Joy $\mathrm{Ngo}^{2}$, Rosa M Ortega ${ }^{3}$, Alicia García ${ }^{2}$, \\ Carmen Pérez-Rodrigo ${ }^{4}$ and Javier Aranceta ${ }^{4}$ \\ 'Department of Clinical Sciences, University of Las Palmas de Gran Canaria, PO Box 550, E-35080 Las Palmas de \\ Gran Canaria, Spain: ${ }^{2}$ Foundation for the Advancement of the Mediterranean Diet, Community Nutrition Research \\ Centre, University of Barcelona Science Park, Barcelona, Spain: ${ }^{3}$ Department of Nutrition, University Complutense of \\ Madrid, Madrid, Spain: ${ }^{4}$ Community Nutrition Unit, Bilbao City Council, Bilbao, Spain
}

\begin{abstract}
Objective: To evaluate dietary habits in Spanish children and adolescents based on a Mediterranean Diet Quality Index tool, which considers certain principles sustaining and challenging traditional healthy Mediterranean dietary patterns.

Design: Observational population-based cross-sectional study. A 16-item Mediterranean Diet Quality Index was included in data gathered for the EnKid study (in which two 24-hour recalls, a quantitative 169-item food-frequency questionnaire and a general questionnaire about socio-economic, demographic and lifestyle items were administered).

Setting: Spain.

Subjects: In total, 3850 children and youths aged 2-24 years residing in Spain. Results: Of the sample, $4.2 \%$ showed very low KIDMED index results, $49.4 \%$ had intermediate values and $46.4 \%$ had high index results. Important geographical differences were seen, with subjects from the Northeast showing the most favourable outcomes ( $52 \%$ with elevated scores vs. $37.5 \%$ of those from the North). Lower percentages of high diet quality were observed in low socio-economic groups, compared with middle and upper income cohorts (42.8\%, 47.6\% and 54.9\%, respectively). Large cities had more positive results and only slight variations were seen for gender and age.

Conclusions: The KIDMED index, the first to evaluate the adequacy of Mediterranean dietary patterns in children and youth, confirms that this collective is undergoing important changes, which makes them a priority target for nutrition interventions. Results challenge certain commonly perceived notions tied to income level, population size and diet quality.
\end{abstract}

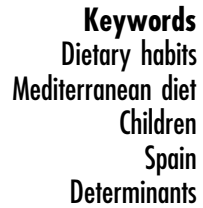

The Mediterranean diet is perhaps one of the healthiest dietary models currently existing, having been corroborated by numerous epidemiological and experimental nutrition studies that show Mediterranean countries benefiting from lower rates of chronic disease morbidity and higher life expectancy. The traditional Mediterranean diet protects against myocardial infarct, certain tumours such as breast, colorectal and prostate, diabetes and other pathologies associated with oxidative stress. Moreover, the Mediterranean diet has been postulated as having a protective role in the development of Alzheimer's disease, certain digestive diseases and even infections, among others. Additionally, it may contribute to reducing complications in various diseases such as the onset of a second myocardial infarct or risk of coronary heart intervention failure, as well as diabetic vascular complications $^{1-3}$.
Despite the fact that the Mediterranean diet concept has gained popularity within the scientific community and the population at large, there is no single Mediterranean diet but rather there are as many Mediterranean eating patterns as there are Mediterranean countries. Furthermore, in addition to defining characteristic food habits, it is important to recognise that not all countries bordering the Mediterranean have preserved the distinctive dietary patterns that were defined in the 1950s and subsequently re-appraised by Professor Ancel Keys ${ }^{4}$. Changes that have occurred in the Mediterranean diet include reduced calorie intake and expenditure, increased consumption of foods with low nutrient density (soft drinks, candy, sweets, etc.) and different food processing techniques (i.e. refining of flour). These factors have impacted upon certain nutritional benefits characteristic of the diet (reduced antioxidant and vitamin intakes, increased 
proportions of saturated fatty acids, decreased fibre consumption, etc.), and reflect changes in cultural values and lifestyles in the Mediterranean. As a result, the legacy the Mediterranean diet represents for the health of its inhabitants has been compromised ${ }^{5,6}$.

Traditional Mediterranean dietary patterns are characterised by elevated intakes of plant foods such as fruits and vegetables, bread and cereals (primarily whole-grain), pulses and nuts. These foods were consumed in season and for the most part fresh, having undergone little or no processing. This enhanced the availability and utilisation of the micronutrients and antioxidants they contained. Olive oil undoubtedly was, and remains to this day, the distinctive dietary element. It may be the only food common to all Mediterranean countries and peoples, crossing ethnic, cultural and religious diversity found throughout mare nostrum. Meat intake was sporadic, only a few times per month, with greater consumption of lamb, poultry, rabbit or fish. Eggs were included in the diet a few times per week. Yoghurt and cheese intakes were abundant, primarily derived from goats and sheep, and milk consumption was less than current intakes. Moreover, moderate wine consumption in combination with an active lifestyle linked to work and transport patterns, completed the characteristic Mediterranean diet model $^{7}$.

Modernisation of society implies that certain sociological changes, such as sedentary lifestyles (due to computers, television, etc.), less time for food preparation and globalisation, are irreversible, and as such, the Mediterranean diet should be adapted to contemporary times without losing its favourable health properties. Following this path, the benefits of the Mediterranean diet should continue to be investigated as well as disseminated to the public, who in fact are the trustees of this value so culturebound that it serves as an identifying mark of Mediterranean populations. Studies of food habits should constitute the basis for planning population-based nutrition programmes. More specifically, nutrition education efforts should be directed towards children to establish healthy eating habits that will have beneficial effects in adulthood. Perhaps children and adolescent populations are those with the most deteriorated Mediterranean diet profile, and thus are worthy of priority attention ${ }^{8}$.

It is important to have access to instruments that allow for the evaluation of dietary habits in children and adolescents, and which also permit the identification of those segments having inadequate intakes. This paper presents the results of the Mediterranean Diet Quality Index for children and adolescents (KIDMED) in Spain.

\section{Material and methods}

The sample analysed, a total of 3850 children and youths aged 2-24 years, was drawn from the EnKid Study population, which has been amply described elsewhere $^{9,10}$.
The development of the KIDMED index was based on principles sustaining Mediterranean dietary patterns as well as those that undermine it. The index ranged from 0 to 12 , and was based on a 16-question test that could be self-administered or conducted by interview (paediatrician, dietitian, etc.). Questions denoting a negative connotation with respect to the Mediterranean diet were assigned a value of -1 , and those with a positive aspect +1 (see Table 1). The sums of the values from the administered test were classified into three levels: (1) $>8$, optimal Mediterranean diet; (2) 4-7, improvement needed to adjust intake to Mediterranean patterns; (3) $\leq 3$, very low diet quality.

Data were evaluated using SPSS for Windows version 10 (SPSS Inc., Chicago, IL, USA). Additionally, descriptive analyses were carried out for the three categories of the KIDMED index by age group (2-14 and 15-24 years), gender, geographic area (Central, Northeast, North, South and Canary Islands, Levant), socio-economic level, mother's educational level and population size.

\section{Results}

Table 2 shows the results of the KIDMED index by age and gender, in which little variation is seen by gender and a slight decline is noted for age. Very low KIDMED index was found for $4.2 \%$ of the sample, $49.4 \%$ demonstrated intermediate values and $46.4 \%$ had high index results. As for geographic area (Fig. 1), the Northeast stands out as having the most favourable values $(52 \%$ with optimal Mediterranean diet quality), whereas the North had the lowest $(37.5 \%)$.

Table 3 reflects the influence of socio-economic level on the Mediterranean diet index. The percentage of the population with a favourable index was $42.8 \%$ in low socio-economic categories versus $54.9 \%$ in the higher

Table 1 KIDMED test to assess the Mediterranean diet quality

\begin{tabular}{ll}
\hline & \multicolumn{1}{c}{ Scoring } \\
\hline+1 & Takes a fruit or fruit juice every day \\
+1 & Has a second fruit every day \\
+1 & Has fresh or cooked vegetables regularly once a day \\
+1 & Has fresh or cooked vegetables more than once a day \\
+1 & Consumes fish regularly (at least $2-3$ times per week) \\
-1 & Goes more than once a week to a fast-food (hamburger) \\
& restaurant \\
+1 & Likes pulses and eats them more than once a week \\
+1 & Consumes pasta or rice almost every day (5 or more times \\
& per week) \\
+1 & Has cereals or grains (bread, etc.) for breakfast \\
+1 & Consumes nuts regularly (at least $2-3$ times per week) \\
+1 & Uses olive oil at home \\
+1 & Skips breakfast \\
-1 & Has a dairy product for breakfast (yoghurt, milk, etc.) \\
+1 & Takes commercially baked goods or pastries for breakfast \\
-1 & Takes sweets and candy several times every day \\
\hline
\end{tabular}

KIDMED - Mediterranean Diet Quality Index in children and adolescents. 
Table 2 KIDMED test and KIDMED index in Spanish children and youths aged 2-24 years by age group and gender $(n=3850)$. EnKid Study (1998-2000)

\begin{tabular}{|c|c|c|c|c|c|c|c|c|c|}
\hline & \multicolumn{3}{|c|}{$2-14$ years } & \multicolumn{3}{|c|}{$15-24$ years } & \multicolumn{3}{|c|}{$2-24$ years } \\
\hline & $\begin{array}{c}\text { Males } \\
(\%)\end{array}$ & $\begin{array}{c}\text { Females } \\
(\%)\end{array}$ & $\begin{array}{c}\text { Total } \\
(\%)\end{array}$ & $\begin{array}{c}\text { Males } \\
(\%)\end{array}$ & $\begin{array}{c}\text { Females } \\
(\%)\end{array}$ & $\begin{array}{c}\text { Total } \\
(\%)\end{array}$ & $\begin{array}{c}\text { Males } \\
(\%)\end{array}$ & $\begin{array}{c}\text { Females } \\
(\%)\end{array}$ & $\begin{array}{c}\text { Total } \\
(\%)\end{array}$ \\
\hline \multicolumn{10}{|l|}{ KIDMED test } \\
\hline Fruit or fruit juice daily & 89.9 & 89.7 & 89.8 & 85.1 & 85.9 & 85.5 & 87.4 & 87.6 & 87.5 \\
\hline Second serving of fruit daily & 63.3 & 62.8 & 63.1 & 55.3 & 59.1 & 57.1 & 59.1 & 60.8 & 59.9 \\
\hline Fresh or cooked vegetables daily & 65.8 & 68.9 & 67.3 & 75.3 & 82.4 & 78.8 & 70.8 & 76.1 & 73.4 \\
\hline Fresh or cooked vegetables $>1 /$ day & 31.7 & 35.3 & 33.4 & 46.4 & 56.8 & 51.5 & 39.4 & 46.8 & 43.0 \\
\hline Regular fish consumption (at least $2-3 /$ week) & 83.3 & 82.5 & 82.9 & 85.5 & 87.4 & 86.4 & 84.5 & 85.1 & 84.4 \\
\hline$>1 /$ week fast-food (hamburger) restaurant & 1.2 & 1.9 & 1.5 & 11.5 & 7.2 & 9.4 & 6.6 & 4.7 & 5.7 \\
\hline Pulses $>1 /$ week & 83.2 & 81.1 & 82.2 & 78.3 & 78.0 & 78.2 & 80.6 & 79.5 & 80.1 \\
\hline Pasta or rice almost daily ( $\geq 5 /$ week) & 37.9 & 37.8 & 37.9 & 36.2 & 26.3 & 31.3 & 37.0 & 31.7 & 34.4 \\
\hline Cereal or cereal product for breakfast & 65.8 & 56.2 & 61.2 & 49.6 & 54.3 & 51.9 & 57.3 & 55.2 & 56.3 \\
\hline Regular nut consumption (at least 2-3/week) & 33.1 & 37.8 & 35.4 & 37.7 & 29.3 & 33.6 & 35.5 & 33.2 & 34.4 \\
\hline Use of olive oil at home & 71.8 & 75.4 & 73.5 & 75.0 & 72.4 & 73.7 & 73.5 & 73.8 & 73.6 \\
\hline No breakfast & 2.5 & 2.8 & 2.6 & 10.2 & 7.3 & 8.8 & 6.5 & 5.2 & 5.9 \\
\hline Dairy product for breakfast & 93.1 & 91.9 & 92.5 & 78.6 & 76.1 & 77.3 & 85.5 & 83.5 & 84.5 \\
\hline Commercially baked goods or pastries for breakfast & 10.9 & 10.2 & 10.6 & 20.0 & 14.3 & 17.2 & 15.7 & 12.4 & 14.1 \\
\hline Two yoghurts and/or $40 \mathrm{~g}$ cheese daily & 54.1 & 48.6 & 51.4 & 50.4 & 45.8 & 48.2 & 52.2 & 47.1 & 49.7 \\
\hline Sweets and candy several times a day & 30.3 & 31.2 & 30.7 & 26.1 & 23.8 & 25.0 & 28.1 & 27.3 & 27.7 \\
\hline \multicolumn{10}{|l|}{ KIDMED index } \\
\hline Poor $(\leq 3)$ & 3.2 & 2.5 & 2.9 & 5.9 & 4.7 & 5.3 & 4.6 & 3.7 & 4.2 \\
\hline Average (4-7) & 47.8 & 49.5 & 48.6 & 50.2 & 49.9 & 50.1 & 49.1 & 49.7 & 49.4 \\
\hline Good ( $\geq 8)$ & 49.0 & 47.9 & 48.5 & 43.9 & 45.4 & 44.6 & 46.3 & 46.6 & 46.4 \\
\hline
\end{tabular}

KIDMED - Mediterranean Diet Quality Index in children and adolescents.

classes. As for mother's educational level, the observed differences were of the same magnitude.

Finally, differences were also observed for index values by resident population size. Large cities were seen to have the most positive results.

\section{Discussion}

Generally speaking, the evaluation of food consumption habits in children and adolescents is more complex than in the adult population as it usually requires the involvement of an adult (customarily the mother) with children under 12. Moreover, greater diet variety as well as between-meal snacking is common during these ages ${ }^{11}$. Globally, in the Spanish Autonomous Communities in which the

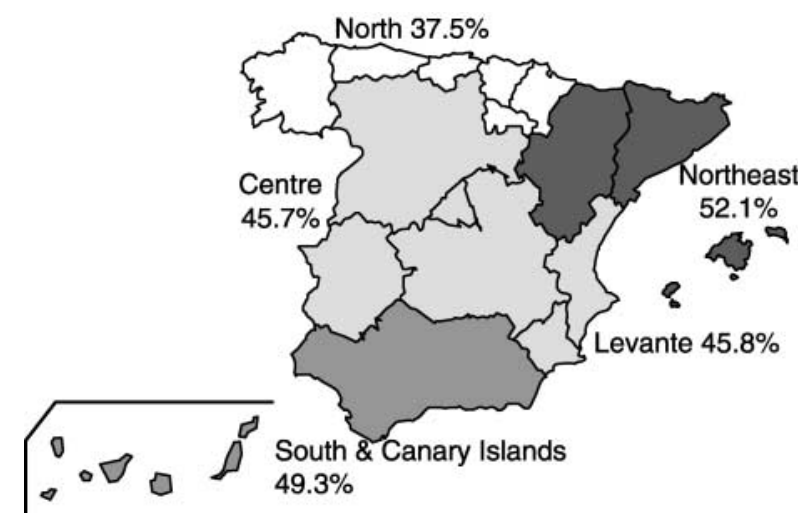

Fig. 1 Spanish population aged 2-24 years with KIDMED index values $\geq 8$ by geographic area, EnKid Study (1998-2000). KIDMED - Mediterranean Diet Quality Index in children and adolescents evaluation of food and nutrient consumption was conducted simultaneously in both adults and youths ${ }^{12,13}$, it is the youngest age groups which show the most compromised intakes and greater abandonment of traditional Mediterranean dietary patterns. This is seen in reduced consumption of fruits, vegetables, pulses and fish within these age groups as compared with adults. These findings, which have also been observed in other countries $^{14}$, raise the question of the Mediterranean diet's capacity to persist into the future ${ }^{15-18}$, as well as the possible increased effects on nutrition-related morbidity and mortality resulting from changes in dietary habits ${ }^{19}$.

Table 3 KIDMED index in Spanish children and youths aged 2-24 years by socio-economic status, mother's educational level and population size. EnKid Study (1998-2000)

\begin{tabular}{lccc}
\hline & \multicolumn{3}{c}{ \% of population with KIDMED index: } \\
\cline { 2 - 4 } & Poor $(\leq 3)$ & Average $(4-7)$ & Good $(\geq 8)$ \\
\hline Socio-economic level & & & \\
Low & 5.1 & 52.0 & 42.8 \\
Medium & 3.8 & 48.5 & 47.6 \\
High & 2.4 & 42.6 & 54.9 \\
Mother's educational level & 4.8 & 52.9 & 42.3 \\
Low & 4.2 & 49.9 & 45.9 \\
Medium & 2.3 & 44.2 & 53.5 \\
High & 4.1 & 51.6 & 44.3 \\
Population size (number of inhabitants) & & 41.2 \\
$\leq 10000$ & 4.0 & 54.7 & 47.6 \\
10 000-50 000 & 5.3 & 47.1 & 52.8 \\
$50000-350000$ & 3.0 & 44.3 & \\
$>350000$ &
\end{tabular}

KIDMED - Mediterranean Diet Quality Index in children and adolescents. 
The EnKid Study, which continues to supply revealing data on the nutrition of Spanish children and adolescents, provides information via this newly developed quality index on the current state and determinants of the Mediterranean diet within this vulnerable collective in Spain. The KIDMED index was inspired by instruments previously developed for adult ${ }^{20}$ and elderly ${ }^{21}$ populations. As such, it constitutes the first index evaluating the adequacy of Mediterranean dietary patterns among the population aged $2-24$ years.

The most relevant results of this first analysis are as follows. The most adverse rating is observed for the North of Spain with the most favourable results seen in the Northeast, which is the region that best preserves Mediterranean dietary patterns in Spain. With respect to the North, it is important to note that the Basque Country shows differential patterns that are more similar to healthy Mediterranean dietary habits than those observed in the other Autonomous Communities bordering the Cantabrian $\mathrm{Sea}^{22}$. Another key finding is the lower index values seen in lower socio-economic or cultural levels. This is due to the fact that, in the lower income categories, less use of olive oil in the home as well as reduced intakes of rice, pasta, fruits and vegetables were observed, despite having the highest intakes of nuts. As such, the commonly held perception that the Mediterranean diet is more economical and thus unaffected by socio-economic standing should be seriously reconsidered.

As a final point, the EnKid Study reveals that, in general, the KIDMED indices are most favourable in larger versus smaller cities. This challenges the assertion that continuity of the traditional Mediterranean diet is greater in smaller cities or towns ${ }^{23}$. The enhanced dietary diversity and availability of fresh products in large urban centres is positively affecting the consumption of healthier foods in Spanish children and adolescents, which will be a subject of further analysis within the EnKid Study in the future.

Special mention should be made about the role of wine in the Mediterranean diet. In the development of the KIDMED index, this was not taken into account given that the consumption of alcoholic beverages is clearly a perturbing element in the nutritional quality of the diets of children and adolescents. Moreover, moderate wine consumption accompanying meals does not constitute a habit commonly seen in these population groups $s^{5,24,25}$.

A large segment of the population is aware of the many benefits of the Mediterranean diet, such as the advantages of olive oil over other dietary fats, the positive effect of moderate wine consumption, or the health benefits of consuming fruits and vegetables, to name a few. However, the question at hand is how to introduce such changes in eating habits. Governments at the regional, national and European level should take steps to prioritise cultivating, raising, producing, transporting and commercialising those foods that constitute the Mediterranean diet food basket: olive oil, fruits and vegetables, fish, cheese and yoghurt, nuts, cereals and wine, among others. Some of these measures are already being carried out; others are not. Families should take on the responsibility of making the healthiest choices at the time of food purchase and preparation at home, or when deciding menu options at a restaurant. The food industry and catering services should also commit to the principles sustaining the Mediterranean diet, the patrimony of our culture and our health, which should not be renounced. Children and adolescents constitute priority targets for action, as the future of the Mediterranean diet rests on the changes that can be effected within these vulnerable collectives ${ }^{4,26}$.

\section{References}

1 Trichopoulou A, Lagiou P. Healthy traditional Mediterranean diet: an expression of culture, history, and lifestyle. Nutrition Reviews 1997; 55: 383-9.

2 Willett WC, Sacks F, Trichopoulou A, Drescher G, Ferro-Luzzi A, Helsing E, et al. Mediterranean diet pyramid: a cultural model for healthy eating. American Journal of Clinical Nutrition 1995; 61(Suppl. 6): 1402S-6S.

3 Serra Majem L. ¿Más beneficios de la dieta mediterránea? Nutrición y Obesidad 2001; 4: 43-6.

4 Keys A. Mediterranean diet and public health: personal reflections. American Journal of Clinical Nutrition 1995; 61(Suppl. 6): 1321S-3S.

5 Serra-Majem L, Santana JF, Salmona E. Dietary habits and nutrient status in Spain. World Review of Nutrition and Dietetics 2000; 87: 127-59.

6 Serra-Majem L, Ferro-Luzzi A, Bellizzi M, Salleras L. Nutrition policies in Mediterranean Europe. Nutrition Reviews 1997; 55(Suppl. 2): S42-57.

7 Serra Majem L, Ribas L. Hábitos alimentarios y consumo de alimentos en España. Dieta mediterránea. In: Serra-Majem L, Aranceta J, Mataix J, eds. Nutrición y Salud Pública. Métodos, Bases Científicas y Aplicaciones. Barcelona: Masson, 1995; 303-10.

8 Serra Majem L, Ngo de la Cruz J, eds. ¿Qué es la Dieta Mediterránea? Barcelona: Nexus Ediciones, 2002; 1-221.

9 Serra Majem L, Aranceta Bartrina J, Ribas Barba L, Pérez Rodrigo C, García Closas R. Estudio enKid: objetivos y metodología. In: Serra-Majem L, Aranceta J, eds. Desayuno y Equilibrio Alimentario. Estudio enKid. Barcelona: Masson, 2000; $1-8$

10 Serra-Majem L, García-Closas R, Ribas L, Pérez-Rodrigo C, Aranceta J. Food patterns of Spanish schoolchildren and adolescents: The enKid Study. Public Health Nutrition 2001; 4: $1433-8$.

11 García Closas R, Serra Majem LI. Encuestas alimentarias en la infancia y adolescencia. Archivos de Pediatría 2000; 51(3): $146-56$

12 Serra Majem L, Ribas L, García Closas R, Ramon JM, Salvador G, Farran A, et al. Llibre Blanc: Avaluació de l'Estat Nutricional de la Població Catalana (1992-93). Barcelona: Departament de Sanitat i Seguretat Social, Generalitat de Catalunya, 1996; 1-252.

13 Serra-Majem L, Ribas Barba L, Armas Navarro A. Equipo investigador de ENCA 1997-98. Encuesta Nutricional de Canarias 1997-98. Ingesta de Energía y Nutrientes y Riesgo de Ingestas Inadecuadas. Archiros Latino-americanos de Nutrición. 2002; 50(Suppl. 1): 75-225.

14 Hercberg S, Preziosi P, Galan P, Deheeger M, Papoz L, Dupin H. Apports nutritionnels d'un échantillon représentatif de la population du Val-de-Marne: III. Les apports en minéraux et 
vitamines. Revue d'Epidémiologie et de Santé Publique 1991; 39: $245-61$.

15 Guerra A, Feldl F, Koletzko B. Fatty acid composition of plasma lipids in healthy Portuguese children: is the Mediterranean diet disappearing? Annals of Nutrition $\mathcal{E}$ Metabolism 2001; 45: 78-81.

16 Greco L, Musmarra F, Franzese C, Auricchio S. Early childhood feeding practices in southern Italy: is the Mediterranean diet becoming obsolete? Study of 450 children aged 6-32 months in Campania, Italy. Cultural Paediatric Association. Acta Paediatrica 1998; 87: 250-6.

17 Cruz JA. Dietary habits and nutritional status in adolescents over Europe - Southern Europe. European Journal of Clinical Nutrition 2000; 54(Suppl. 1): S29-35.

18 Avellone G, Di Garbo V, Panno AV, Cordova R, Abruzzese G, Rotolo G, et al. Cardiovascular risk factors and dietary habits in secondary school children in southern Italy. International Angiology 1994; 13: 148-53.

19 Serra-Majem L, Helsing E, eds. Changing patterns of fat intake in Mediterranean countries. European Journal of Clinical Nutrition 1993; 47(Suppl. 1): S1-100.

20 Alberti-Fidanza A, Fidanza F. Mediterranean Adequacy Index of Italian diets. Public Health Nutrition 2004; 7: 937-41.
21 Trichopoulou A, Kouris-Blazos A, Wahlqvist ML, Gnardellis C, Lagiou P, Polychronopoulos E, et al. Diet and overall survival in elderly people. British Medical Journal 1995; 311(7018): 1457-60.

22 Aranceta Bartrina J, Pérez Rodrigo C. Consumo de Alimentos y Estado Nutricional de la Población de Bilbao. Guías Nutricionales para la Población Escolar. Bilbao: Área de Salud y Consumo, Excmo, Ayuntamiento de Bilbao, 1996.

23 Petridou E, Malamou $\mathrm{H}$, Doxiadis S, Pantelakis S, Kanellopoulou G, Toupadaki N, et al. Blood lipids in Greek adolescents and their relation to diet, obesity, and socioeconomic factors. Annals of Epidemiology 1995; 5: 286-91.

24 De Lorgeril M, Salen P. Wine ethanol, platelets, and Mediterranean diet. Lancet 1999; 353: 1067.

25 De la Torre-Boronat MC. Scientific basis for the health benefits of the Mediterranean diet. Drugs under Experimental and Clinical Research 1999; 25: 155-61.

26 Birch LL, Fisher JO. Development of eating behaviours among children and adolescents. Pediatrics 1998; 101: 539-49. 